\title{
Recognizing Human Activities Based on Wearable Inertial Measurements - Methods and Applications
}

\author{
Pekka Siirtola \\ Computer Science and Engineering Department, University of Oulu, Oulu, Finland
}

\begin{abstract}
On April 10 of 2015 Pekka Siirtola defended his PhD thesis, called "Recognizing Human Activities Based on Wearable Inertial Measurements - Methods and Applications" [1]. The thesis was supervised by Professor Juha Röning and preeximined by Associate Professors Ulf Johansson from University of Borås, Sweden, and Daniel Roggen from University of Sussex, United Kingdom. Pekka Siirtola successfully defended his thesis against his opponent Professor Barbara Hammer from University of Bielefeld, Germany. This publicly open defence was held in Auditorium TS101 at University of Oulu, Finland.
\end{abstract}

Keywords - Activity recognition, inertial sensors, pattern recognition

\section{INTRODUCTION}

$\mathrm{I}$ NERTIAL sensors are devices that measure movement, and therefore, when they are attached to a human body, they can be used to measure human movements. In the doctoral thesis "Recognizing Human Activities Based on Wearable Inertial Measurements - Methods and Applications" by Pekka Siirtola, data from these sensors are studied to recognize human activities user-independently. This is possible if the following two hypotheses are valid: firstly, as human movements are dissimilar between activities, also inertial sensor data between activities is so different that this data can be used to recognize activities. Secondly, while movements and inertial data are dissimilar between activities, they are so similar when different persons are performing the same activity that they can be recognized as the same activity. In the thesis, pattern recognition -based solutions are applied to inertial data to find these dissimilarities and similarities, and therefore, to build models to recognize activities user-independently.

The thesis is a compilation of six original publications ([2] - [7]) and it studies activity recognition in two contexts: daily activity recognition using mobile phones, and activity recognition in industrial context. Both of these contexts have special requirements and these are considered in the presented solutions. Mobile phones are optimal devices to measure daily activity: they include a wide range of useful sensors to detect activities, and people carry them with them most of the time. On the other hand, the usage of mobile phones in activity recognition includes several challenges; for instance, a person can carry a phone in any orientation, and there are hundreds of smartphone models, and each of them has specific hardware and software. In addition, in order to achieve highest possible recognition rates, all sensors need to be calibrated before they are used no matter if the used sensor is an inertial sensor or a camera [8]. Activity recognition using the inertial sensors of mobile phones are studied in original publications [2] and [3]. Moreover, as battery life is always as issue with smartphones, techniques to lighten the classification process are proposed (studied in original publications [4] and [5]). Industrial context is different from daily activity context: when daily activities are recognized, occasional misclassifications may disturb the user, but they do not cause any other type of harm. This is not the case when activities are recognized in industrial context and the purpose is to recognize if the assembly line worker has performed tasks correctly. In this case, false classifications may be much more harmful. Solutions to these challenges are presented in the thesis (studied in original publications [6] and [7]).

The solutions introduced in the thesis are applied to activity recognition data sets. However, as the basic idea of the activity recognition problem is the same as in many other pattern recognition procedures, most of the solutions can be applied to any pattern recognition problem, especially to ones where time series data is studied.

\section{REFERENCES}

[1] Siirtola, Pekka (2015), "Recognizing human activities based on wearable inertial measurements: methods and applications", Department of Computer Science and Engineering, University of Oulu, April, Acta Universitatis Ouluensis C 524.

[2] Siirtola P \& Röning J (2012), "Recognizing Human Activities Userindependently on Smartphones Based on Accelerometer Data", International Journal of Interactive Multimedia and Artificial Intelligence (1), 5 (June 2012), 38-45.

[3] Siirtola P \& Röning J (2013), "Ready-to-Use Activity Recognition for Smartphones". IEEE Symposium on Computational Intelligence and Data Mining (CIDM 2013), 16-19 April 2013, 59-64.

[4] Siirtola P, Laurinen P, Röning J \& Kinnunen H (2011), "Efficient Accelerometer-Based Swimming Exercise Trackino" IF.F.F. Svmnnsium on Computational Intelligence and L $\quad$ DOI: 10.9781/ijimai.2015.336 $15,156-161$. 
[5] Siirtola P, Koskimäki H \& Röning J (2011), "Periodic Quick Test for Classifying Long-term Activities", IEEE Symposium on Computational Intelligence and Data Mining (CIDM 2011), April 11-15, 135--140.

[6] Siirtola P, Koskimäki H, Laurinen P, Huikari V \& Röning J (2011), "Improving the Classification Accuracy of Streaming Data Using SAX Similarity Features", Pattern Recogn. Lett. 32, 13 (October 2011), 16591668.

[7] Koskimäki H, Huikari V, Siirtola P \& Röning J (2013), "Behavior modelling in industrial assembly lines using a wrist-worn inertial measurement unit", Journal of Ambient Intelligence and Humanized Computing: Volume 4, Issue 2 (2013), Page 187-194.

[8] Pacheco A, Bolivar-Baron H, Gonzalez-Crespo R, \& Pascual-Espada J (2014), "Reconstruction of High Resolution 3D Objects from Incomplete Images and 3D Information", International Journal of Interactive Multimedia and Artificial Intelligence, vol. 2, issue Regular Issue, no. 6, pp. 7-16.

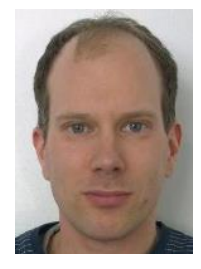

Pekka Siirtola received his M.Sc. degree in Mathematics in February 2006 and Dr.Tech degree in April 2015, both from University of Oulu, Oulu, Finland. Currently, he is working as a post-doc at the Computer Science and Engineering Department at University of Oulu. His research interests include machine-learning aspects of signal shapes and, especially, activity recognition based on inertial measurements. 